\title{
Estudio piloto in vitro para determinar la precisión del ARCUSdigma en la determinación de posiciones condilares
}

\section{In vitro pilot study to determine the accuracy of the ARCUSdigma to determine condylar positions}

Arroyo Cruz G*, Orozco Varo A*, Ventura de la Torre J**, Cañadas Rodríguez D***, Jiménez-Castellanos Ballesteros $\mathrm{E}^{* * * * *}$

\section{RESUMEN}

En este trabajo pretendemos determinar la precisión, entendida como el grado de concordancia de las medidas entre si, de un pantógrafo electrónico (ARCUSdigma) en la determinación de dos posiciones condilares seleccionadas, sobre una muestra constituida por los modelos de siete pacientes montados en articulador semiajustable. Tras analizar los resultados obtenidos y teniendo en cuenta las limitaciones con que cuenta el estudio, podemos concluir que dicho instrumento presenta una alta precisión a la hora de realizar tales determinaciones.

Palabras clave: Pantografía, posición condilar.

\section{SUMMARY}

In this work we seek to determine the accuracy (it is the concordance of the measures) of an electronic pantograph (ARCUSdigma) to determine two condylar positions. We have a sample constituted by the models of seven patients mounted in a semiajustable articulator. In spite of the limitations of this study, the results indicate that the instrument has a high precision for these determinations.

Key words: Pantographic, condylar position.

Fecha de recepción: Febrero 2008.

Aceptado para publicación: Marzo 2008.

* Colaboradora Clínica de Prótesis Estomatológica y Oclusión. Facultad de Odontología de Sevilla.

** Profesor Asociado de Prótesis Estomatológica y Oclusión. Facultad de Odontología de Sevilla.

*** Profesor Titular de Prótesis Estomatológica y Oclusión. Facultad de Odontología de Sevilla.

**** Catedrático de Prótesis Estomatológica y Oclusión. Facultad de Odontología de Sevilla.

Arroyo Cruz G, Orozco Varo A, Ventura de la Torre J, Cañadas Rodríguez D, Jiménez-Castellanos Ballesteros E. Estudio piloto in vitro para determinar la precisión del ARCUSdigma en la determinación de posiciones condilares. Av. Odontoestomatol 2008; 24 (5): 333-341.

\section{INTRODUCCIÓN}

En oclusión se conoce con el nombre de Instrumentación (1) al conjunto de aparatos y medios técnicos que permiten, por una parte, registrar y analizar los desplazamientos condilomeniscales y, por otro, reproducirlos (articuladores).
Actualmente, las técnicas de registro y análisis de la dinámica mandibular son múltiples y variadas, encontrándose entre ellas la pantografía.

La pantografía se define como la reproducción a distancia de un movimiento determinado (2), permitiendo en el campo de la oclusión, registrar las tra- 
yectorias y movimientos mandibulares en los tres planos del espacio, a nivel condilar e incisal.

Una de sus principales funciones es la programación de articuladores totalmente ajustables, si bien juega también un papel fundamental en la evaluación y el diagnóstico de la coordinación neuromuscular, de la calidad del movimiento mandibular y de la patología articular específica del paciente (1).

El Gnathograph (3) presentado por Beverly B. McCollum y Charles E, Stuart se puede considerar como el primer pantógrafo mecánico de concepción actual, "el primer dispositivo satisfactorio para registro" como se diría de él cuando se presentó definitivamente, en 1937.

En 1968, Niles Guichet desarrolla el modelo de articulador Denar D4A con su pantógrafo mecánico incorporado, siendo posteriormente perfeccionado por la Denar Corporation con el modelo Denar D5A y su sistema Pantronic para el registro de los movimientos mandibulares (4).

Otros sistemas pantográficos asistidos por ordenador han ido apareciendo en el mercado como el sistema Rosy $(5,6)$, el Artex, el Condylocomp LR2 (7) y LR3 (8) y ARCUSdigma (9), que será objeto de nuestro estudio.

Se trata de un pantógrafo electrónico introducido casa alemana Kavo (KaVo elektrotechnisches, Werk, GMBH, Leutkitch, Germany) en 2002, cuyo funcionamiento está basado en la emisión-recepción de ultrasonidos. Entre las distintas utilidades que presenta, está el análisis electrónico de posición condilar. Dicha opción permite comparar posiciones condilares valorando la discrepancia existente entre ellas, cualitativa y cuantitativamente.

\section{OBJETIVO}

El objetivo de nuestro estudio ha sido determinar la precisión del ARCUSdigma de Kavo en la determinación de dos posiciones condilares elegidas, en dos momentos distanciados en el tiempo, sobre modelos montados en un articulador semiajustable.

Por "precisión" (10) entendemos el grado de concordancia de las medidas entre si, sin determinar en principio que estos valores obtenidos coincidan o no con el valor real.

\section{MATERIAL Y MÉTODO}

\section{Selección de la muestra}

La muestra de nuestro estudio piloto la han constituido siete modelos de escayola tomados a alumnos de la Facultad de odontología de Sevilla. Los criterios de selección que hemos establecido son los siguientes:

1. Máxima intercuspidación estable.

2. Ausencia de sintomatología objetiva o subjetiva de patología temporomandibular.

3. Dentición definitiva con ausencia de no más de 4 dientes, ni más de uno por hemiarcada, exceptuando los terceros molares.

4. Ausencia de deformidades craneofaciales evidentes.

5. No ser portador de prótesis.

La selección de la muestra la realizó un operador previamente entrenado, siguiendo un protocolo de exploración elaborado por la unidad docente de Prótesis y Oclusión de la Facultad de Odontología de Sevilla basándose en el de Dworkin y LeResche (11). Los datos de dicha exploración quedaron recogidos en la correspondiente historia clínica.

Toma de impresiones, obtención de los modelos, transferencia de éstos al articulador y preparación de las fijaciones del emisor de ultrasonidos del kavo ARCUSdigma

Una vez seleccionada la muestra, se procedió a la toma de impresiones con alginato (VIVAL de la casa Ivoclar Vivadent AG, FL-9494 Schaan/Liechtenstein), mediante cubetas metálicas prefabricadas estandarizadas para sujetos dentados (ÁSA Dental S.p.a. Via Valenzana, 4, I-55050. Bozzano, LU, Italy), y se positivaron con escayola piedra mejorada (Kromotypo type 4, Lascod. Fierenze. Italy).

Los modelos obtenidos se trasfirieron a un articulador semiajustable Quick Perfect (de la casa francesa F.A.G. Detaire). El modelo superior se relacionó con 
el articulador mediante el correspondiente arco facial de transferencia. Por su parte, el modelo inferior se relacionó con el superior en posición de máxima intercuspidación, sin registros interpuestos.

Para fijar ambos modelos al articulador se utilizó escayola de fraguado rápido (Snow white de la casa Kerr).

Sobre el modelo inferior construimos, con resina de la marca Duralay, la fijación para el emisor de ultrasonidos del ARCUSdigma (Fig 1). La horquilla metálica se adaptó a la curvatura de la arcada dentaria inferior, por vestibular del grupo incisivo y premolar. En casos de sobremordida intensa, la resina se adaptará por vestibular de los sectores posteriores para evitar interferencias durante la máxima intercuspidación.

\section{Instrumentos de medida}

El método empleado ha seguido las instrucciones de uso del fabricante, pero al tratarse de un estudio in Vitro, el arco facial y los receptores (fig. 2) de que consta el ARCUSdigma se posicionaron sobre el articulador semiajustable Quick-Perfect (fig. 3) al que previamente acoplamos una serie de aditamentos que garantizaran la estabilidad de dicho arco:

- Fijamos dos barras en la parte posterior de la rama superior del articulador, sobre los cajetines, con cianocrilato y reforzadas con resina acrílica fotopolimerizable, de tal forma que constituyeran una base sólida sobre la que apoyar el arco, lo que boca sería el apoyo que proporciona la parte superior y posterior del pabellón auditivo (fig. 4).

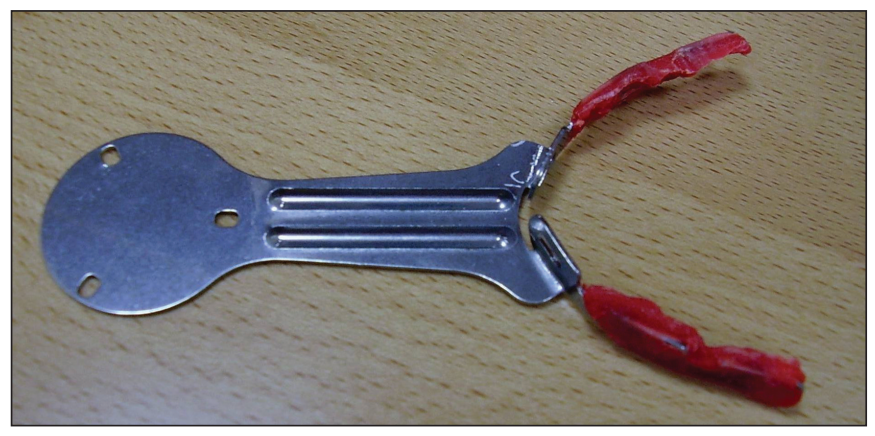

Fig. 1. Fijación para el emisor del ARCUSdigma.

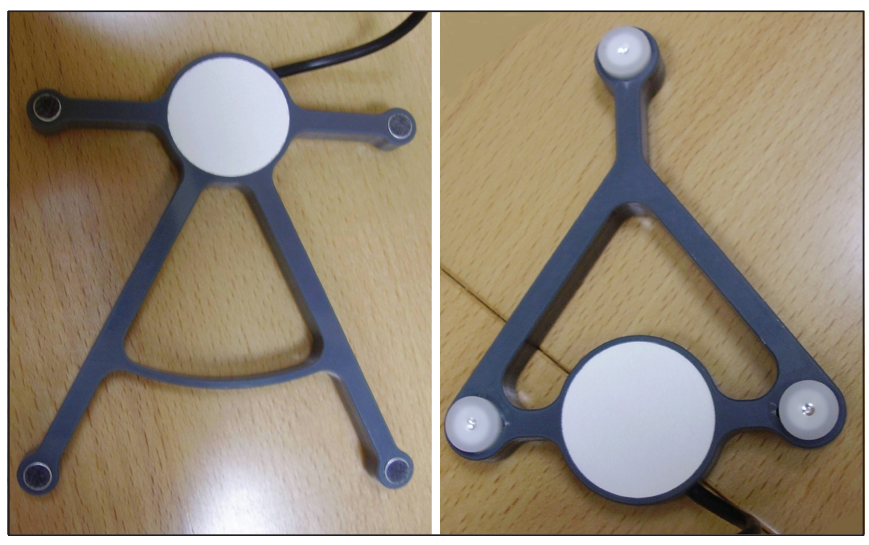

Fig. 2. Receptor y emisor del ARCUSdigma.

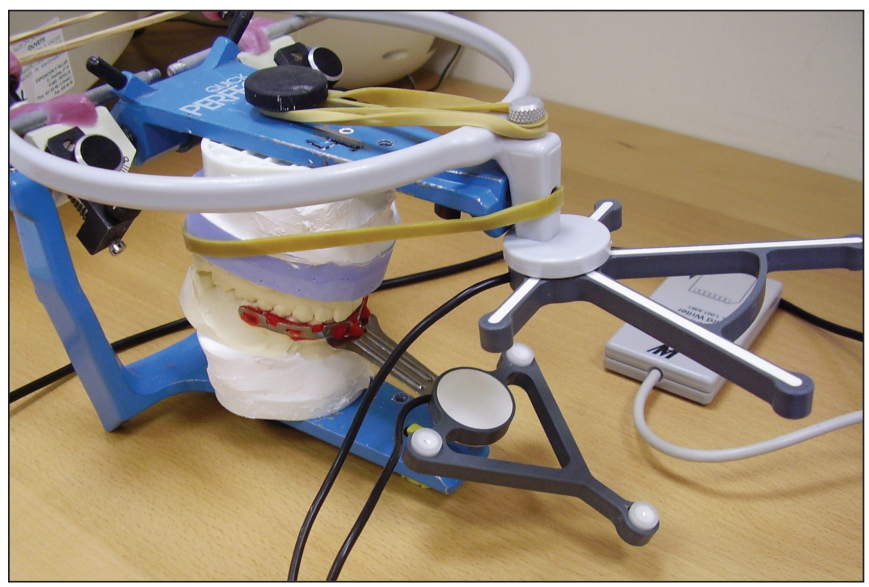

Fig. 3. ARCUSdigma colocado sobre el Quick Perfect.

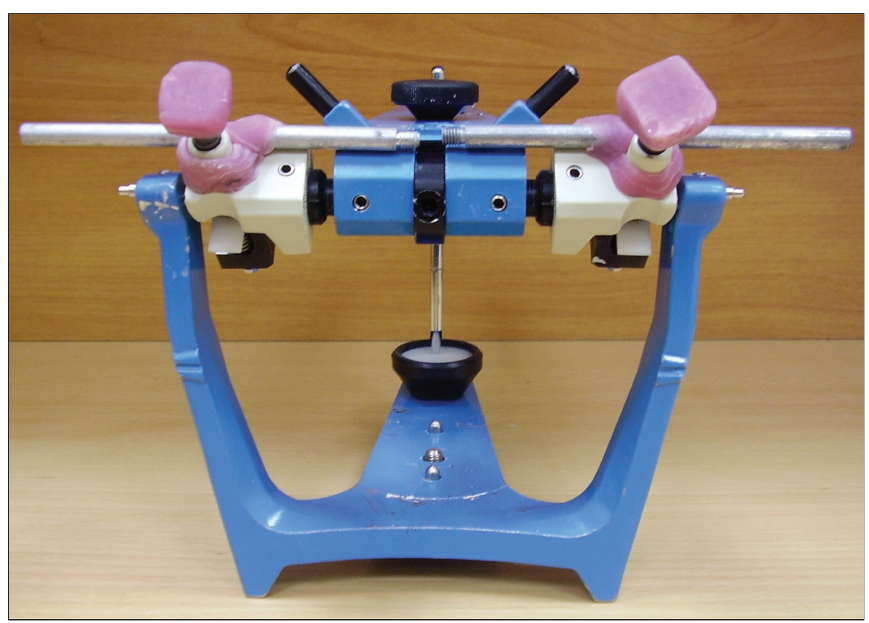

Fig. 4. Modificaciones realizadas en el Quick Perfect. 
- Confeccionamos unos aditamentos que fijamos a los tornillos de protrusiva para aseguran transversalmente la estabilidad del arco (la función que en boca haría la propia fascies del paciente) (fig 4).

- Una cinta elástica unió la parte posterior de arco y otra la zona anterior al tornillo que fija la pletina superior. Esta última haría la función del nasión en el paciente real.

- La horquilla inferior del ARCUSdigma se fijó mediante cianocrilato sobre la superficie vestibular de los dientes anteroinferiores del modelo, de tal manera que no interfiera con ninguna posición ni movimiento.

\section{Mediciones con el epa-test del ARCUSdigma}

Una vez expuesto el contexto en el que realizaremos las mediciones, procedemos a detallar la sistemática que hemos seguido para llevarlas a cabo:

1. Realizamos dos secuencias de 10 mediciones para comprobar la exactitud del ARCUSdigma a la hora de reproducir la posición de Máxima intercuspidación (MI) (Fig. 5).

2. Realizar dos secuencias de 10 mediciones para comprobar la exactitud del ARCUSdigma a la hora de reproducir una protrusión de $5 \mathrm{~mm}$, predeterminada en el tornillo telescópico del articulador.

Un único operador, previamente adiestrado, realizó las mediciones con el ARCUSdigma repitiendo el procedimiento a los pocos días para comparar los resultados.

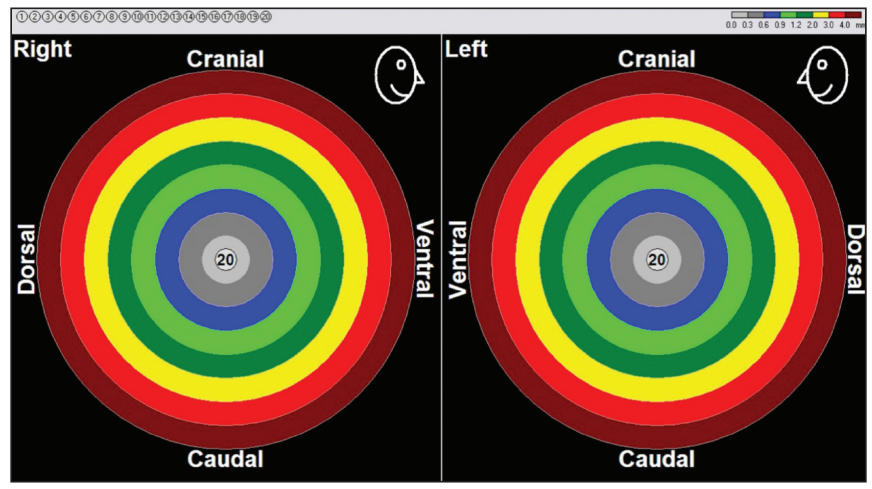

Fig. 5. Representación gráfica de las mediciones en la pantalla de ordenador del ARCUSdigma. Muestra la coincidencia de dos secuencias de 10 mediciones.

\section{RESULTADOS}

Hemos realizado un análisis porcentual de los resultados obtenidos en cada uno de los siete casos, para cada una de las dos posiciones que hemos pretendido registrar. Dicho análisis se ha hecho en base a la coincidencia o no de las sucesivas mediciones con respecto a las de referencia.

\section{Resultados en máxima intercuspidación}

En cada caso se han realizado un total de 40 mediciones (dos secuencias de 20) para cada articulación y con representación en los tres planos del espacio. Si las 40 mediciones eran coincidente con las de referencia establecemos que la precisión del ARCUSdigma en dicho procedimiento es del $100 \%$, si no fuera así establecemos una regla de tres para hallar el porcentaje que corresponde a cada caso (por ejemplo, si 40 es $100 \%$, 39 es 97,5\%). En la tabla 1 se recogen la precisión obtenida en cada una de las mediciones.

\section{Resultados en protrusiva de $5 \mathrm{~mm}$}

Al igual que en el caso anterior se han realizado un total de 40 mediciones (dos secuencias de 20) para cada articulación y con representación en los tres planos del espacio. Si las 40 mediciones eran coincidente con las de referencia establecemos que la precisión del ARCUSdigma en dicho procedimiento es del $100 \%$. En la tabla 2 se recogen la precisión obtenida en cada una de las mediciones.

\section{Media de resultados en máxima intercuspidación}

En la tabla 3 se recoge la media de las mediciones de MI, en cada articulación y en cada plano de espacio, así como la total por articulaciones.

\section{Media de resultados en protrusiva de $5 \mathrm{~mm}$}

En la tabla 4 se recoge la media de las mediciones de protrusiva de $5 \mathrm{~mm}$, en cada articulación y en cada plano de espacio, así como la total por articulaciones. 
TABLA 1

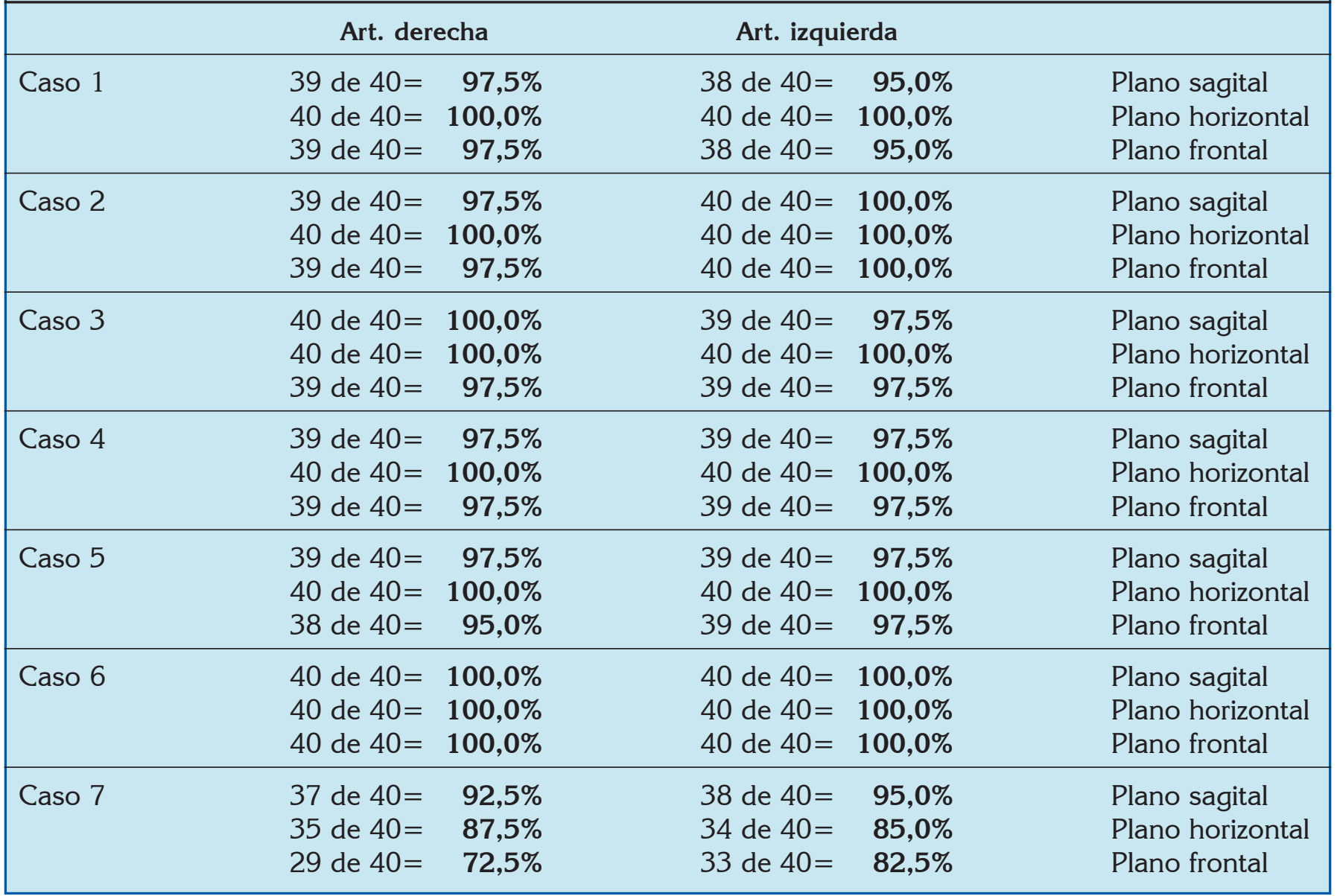

De los 3.360 datos que hemos manejado en nuestro estudio, el ARCUSdigma se muestra coincidente en 3.223 de ellos, luego podemos concluir que a la luz de los resultados obtenidos muestra una precisión del $95,92 \%$.

\section{DISCUSIÓN}

En nuestro trabajo, a pesar de ser el ARCUSdigma un instrumento diseñado para usarse directamente sobre el paciente, hemos optado por realizar un primer estudio piloto in vitro, para tener la certeza de que en un ambiente controlado se comporta de manera precisa. Estableciendo así el punto de partida necesario a partir del cual, en un futuro, usarlo in vivo.
A la luz de los resultados obtenidos y pese a lo reducido de la muestra, al tratarse de un estudio piloto, el ARCUSdigma resulta un método preciso para determinar in vitro las posiciones condilares elegidas.

Los estudios in vitro con pantógrafos son un proceder habitual en la investigación científica, encontrando en la literatura trabajos como el de Anderson y colaboradores (12) que evalúa la fiabilidad y exactitud de un pantógrafo electrónico (Pantronic de Denar) mediante el registro de los movimientos de un articulador totalmente ajustable, el Denar D5-A con parámetros condilares conocidos. Concluyen que el Pantronic es un método fiable en la determinación de la inclinación de la trayectoria condilar (ITC), el Shift inmediato y el progresivo. Pelletier y 
AVANCES EN ODONTOESTOMATOLOGÍA

Vol. 24 - Núm. 5 - 2008

TABLA 2

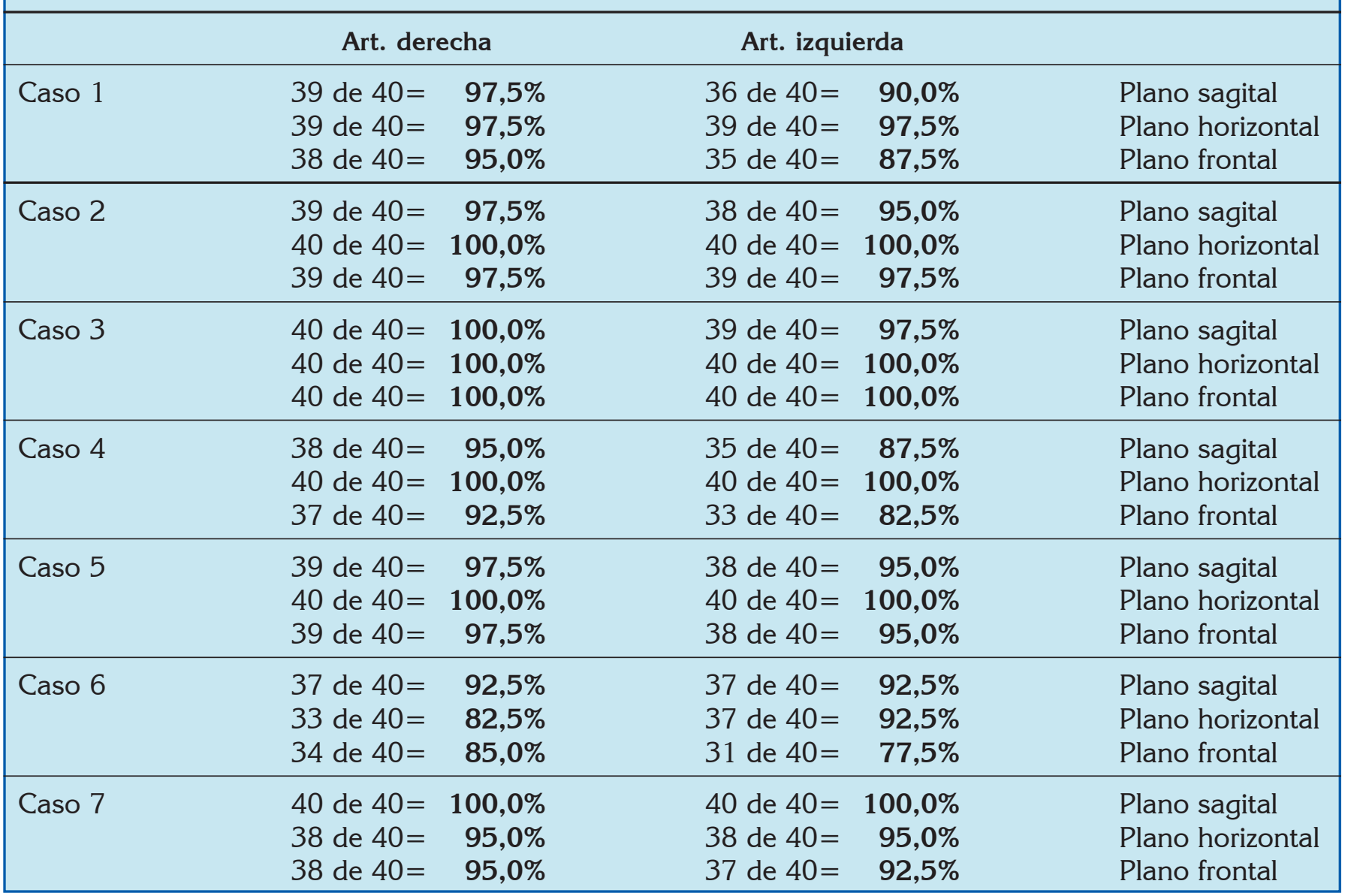

TABLA 3

Art. derecha

Plano sagital

Plano horizontal

Plano frontal

TOTAL
273 de $280=97,50 \%$

275 de $280=98,21 \%$

263 de $280=93,92 \%$

811 de $840=96,54 \%$
Art. izquierda

273 de $280=97,50 \%$

274 de $280=97,85 \%$

268 de $280=95,35 \%$

815 de $840=97,02 \%$

TABLA 4

Art. derecha

\section{Plano sagital}

Plano horizontal

Plano frontal

TOTAL
272 de $280=97,14 \%$

270 de $280=96,42 \%$

265 de $280=94,64 \%$

807 de $840=96,07 \%$
Art. izquierda

263 de $280=93,93 \%$

274 de $280=97,85 \%$

253 de $280=90,35 \%$

790 de $840=94,04 \%$ 
Arroyo Cruz G, Orozco Varo A, Ventura de la Torre J, Cañadas Rodríguez D, Jiménez-Castellanos Ballesteros E. Estudio piloto in vitro para determinar la precisión del ARCUSdigma en la determinación de posiciones condilares

colaboradores (13) en su estudio comparan la fiabilidad y exactitud en la determinación de la ITC entre el pantógrafo mecánico y el electrónico de la casa Denar, resultando éste último superior y determinando además que los sistemas pantográficos en general proporcionan más exactitud y fiabilidad en la determinación de la ITC que otras técnicas de registro. Shulte y colaboradores (14) utilizan también un pantógrafo electrónico para determinar, tridimensionalmente sobre el articulador Denar D5-A los cambios en las trayectorias de las cúspides de un molar al cambiar las guías condíleas y la anterior. Tenemos por otro lado trabajos como el de Cellar y Tamaki (15) que evalúan la exactitud del Cardiax Compact al medir la ITC y el ángulo de Bennet sobre un articulador Artez, obteniéndose con él, resultados clínicamente aceptables. Es el también el Cardiax Compact el pantógrafo que utilizan Chang y colaboradores (16) en su trabajo, sobre cinco articuladores (distintos Denar D5A, Denar Mark II, Whip Mix 8500, Hanau Modular y Panadent PCH) calculando la ITC. Opinan que es mejor realizar dicho procedimiento sobre articuladores y no sobre pacientes para poder conocer el valor concreto y compararlo con el resultado obtenido por el instrumento. Concluyen que el Cardiax Compact de Denar es un método fiable y válido obteniéndose los mejores resultados a $10 \mathrm{~mm}$ de desplazamiento condilar, por lo que recomiendan programar el articulador con los resultados obtenidos con dicho desplazamiento.

En un estudio reciente de Baker y colaboradores (17) encontramos que utilizan in vitro el ARCUSdigma planteando como objetivos por un lado examinar la habilidad de dicho pantógrafo para registrar valores condilares preseleccionados en el articulador Protar y en segundo lugar usarlo para regular los articuladores Protar y Denar y realizar con esos valores trazados pantográficos con el pantógrafo mecánico del Denar y compararlos. Obtienen como conclusiones que el ARCUSdigma es capaz de registrar con precisión la ITC, pero no el shift inmediato, el progresivo o el ángulo shift. Esto último puede tener relevancia clínica ya que estos parámetros condicionan el recorrido de las cúspides de trabajo y no trabajo.

Pese a tratarse de estudios in Vitro y concretamente el de Baker, usar el mismo instrumento que noso- tros, no es posible una comparativa entre nuestros resultados y los encontrados en la literatura pues todos estos estudios toman como objeto de estudio parámetros guía para programar el articulador como son la ITC, el ángulo de Bennet, etc, y no la posición condilar en una relación dental estática como puede ser la de máxima intercuspidación.

Determinar la posición en que su ubican los cóndilos en la articulación temporomandibular ha sido y sigue siendo un tema de vital trascendencia en oclusión. Desde que la escuela gnatológica de McCollum tomara una referencia condilar (relación céntrica) como punto de partida para sus rehabilitaciones, dicha posición ha tenido un papel clave en campos como el de la prostodoncia y ortodoncia, siendo muchos los autores que abogan por ello (18-22).

El binomio oclusión-posición condilar, es decir, como la posición condilar está determinada por la oclusión (18) y como la morfología oclusal, con sus elevaciones y depresiones, no es un mero accidente anatómico, sino que está relacionada con las características temporomandibulares es un hecho por todos aceptado (23). Sin embargo el papel que este binomio tiene en los desórdenes temporomandibulares esta de nuevo sujeto a controversia (21).

Vemos pues como existe un halo de misterio y controversia envolviendo estos aspectos relacionados como la posición condilar. Por ello instrumentos como el ARCUSdigma de Kavo que permiten el estudio de este parámetro deben ser tenidos en cual y despertar nuestro interés.

Esto es algo que hasta el momento se ha venido haciendo, no con sistemas pantográficos sino con aditamento que acoplados a un articulador en cuestión permite tal función. Ese es el caso del MPI que es la parte superior modificada de un articulador del sistema SAM. Son numerosos los estudios que hallamos en la literatura en los que se utiliza tal instrumento. Tenemos el de Wood y Korne (24) en el que en una muestra de 39 sujetos plantean entre otros objetivos determinar la reproducibilidad del MPI, concluyendo que resulta un instrumento altamente reproducible. Alexander y colaboradores (25) en una muestra de 28 sujetos se plantean determinar la 
existencia de distintas posiciones mandibulares utilizando para ello entre otros procedimientos el MPI. Bujaldón (26) valora entre otros parámetros la discrepancia entre dos posiciones condilares, en 124 pacientes usando el MPI.

El articulador Panadent dispone también de un indicador de posición condilar, el CPI. Al igual que con el MPI también encontramos en la literatura estudios que hacen uso de dicho instrumento, fundamentalmente para comparar distintas posiciones condilares. Condray (27) presenta un trabajo sobre 596 pacientes en lo que evalúa la naturaleza del los desplazamientos condilares entre relación céntrica $(\mathrm{RC})$ y oclusión céntrica $(\mathrm{OC})$ utilizando el CPI. Wood y Elliot (28) determinan, usando el CPI, la dirección del desplazamiento céntrico en una muestra de 39 sujetos. Es también el desplazamiento céntrico el parámetro que mide Crawford (18) en su estudio sobre 60 sujetos, parte de los cuales habían sido rehabilitados con una concepción gnatológica y otros que constituían el grupo control. Utiliza para cuantificar dicho desplazamiento el CPI. Karl y Foley (29) utilizan en su estudio de 40 sujetos el CPI para investigar las diferencias entre registros de RC antes y después de usar un jig.

Hicks y Wood (30) evalúan la dirección del movimiento condilar de RC a OC usando para ello tanto el MPI como el CPI sobre una muestra de 37 sujetos.

La determinación de posiciones condilares que permite el ARCUSdigma presenta frente a estos instrumentos antes mencionados una ventaja clave, la inmediatez del procedimiento, ahorrando algo cada día más escaso en nuestra sociedad, el tiempo. Consideramos pues fundamental ahondar en este procedimiento que como comentamos anteriormente será objeto de nuestros futuros estudios.

\section{CONCLUSIÓN}

Teniendo en cuenta las limitaciones, ya mencionadas, de nuestro estudio, podemos concluir en base a los resultados obtenidos que el ARCUSdigma presenta una precisión del 95,92\% en la determinación de las posiciones condilares seleccionadas para el estudio.

\section{BIBLIOGRAFÍA}

1. El Manual de Odontología. Barcelona: MASSON; 1995.

2. Sencherman de Savdie G, Echeverri E. Neurofisiología de la oclusión. Bogotá: Ediciones Monserrate LTDA; 1995.

3. Starcke E. The history of articulators: from facebows to the gnathograph, a brief history of early devices developed for recording condylar movement: part II. J Prosthodont 2002;11:5362.

4. Malone WFP, Koth DL, Cavatosi E, Kaiser DA, Morgano SM. Tylman's. Teoría y práctica en prostodoncia fija. Caracas: Actualidad Médico Odontológica Latinoamérica; 1991.

5. Edinger DH. Accuracy of a robotic system for the reproduction of condylar movements: a preliminary report. Quintessence Int. 2004;35: 519-23.

6. Edinger DH. Instrumental functional diagnostics with the ROSY simulator.Int J Comput Dent. 2003;6:37-49.

7. Ott $\mathrm{KH}$, Olavarria LE, Bose M. Studies on the reproducibility of recordings obtained with the Condylocomp LR 2.Dtsch Zahnarztl Z. 1991; 46:596-8.

8. Olthoff LW, Van Der Zel JM, De Ruiter WJ, Vlaar ST, Bosman F. Computer modeling of occlusal surfaces of posterior teeth with the CICERO CAD/CAM system. J Prosthet Dent. 2000; 84:154-62.

\section{Instrucciones del ARCUSdigma.}

10. Calatayud J, Martín G. Bioestadística en la investigación odontológica. Madrid: Pues SL; 2002.

11. Dworkin SF, LeResche L. Research Diagnostic Criteria for Temporomandibular Disorders: Review, criteria, examinations and specifications, critique. J Craniomandib Disord 1992;6:301-55. 
12. Anderson GC, Schulte JK, Arnold TG. An in vitro study of an electronic pantograph. J Prosthet Dent. 1987;57:577-80.

13. Pelletier LB; Campbell SD: Comparison of condylar control settings using three methods: a bench study. J Prosthet Dent 1991;66:193-200.

14. Schulte JK, Wang SH, Erdman AG, Anderson GC. Three dimensional analysis of cusp travel during a nonworking mandibular movement. J Prosthet Dent 1985;53:839-43.

15. Celar AG, Tamaki K. Accuracy of recording horizontal condylar inclination and Bennett angle with the Cadiax compact ${ }^{\circledR}$. J Ora Rehabil. 2002;29: 1076-81.

16. Chang WS, Romberg E, Driscoll CF, Tabacco MJ. An in vitro evaluation of the reliability and validity of an electronic pantograph by testing with five different articulators. J Prosthet Dent 2004;92:839.

17. Baker PJ, Setchell DJ, Tredwin CJ. Reproduction ofarticulator settings and movements with an ultrasonic jaw movement recorder. Eur J Prosthodont Restor Dent. 2006;14:55-62.

18. Crawford SD. Condylar axis position, as determined by the occlusion and mesured by the CPI instrument, and signs and symptoms of temporomandibular dysfunction. Angle Orthod 1999; 69:103-14.

19. Espinar E. Férulas oclusales como principio de obtención del diagnóstico en relación céntrica en ortodoncia. Manejo clínico. Rev Esp Ortod. 2003;33:41-9.

20. Williamson EH. Concepto de la posición condilar fisiológica. Rev Esp Ortod 1981;11:21-8.

21. González Sequeros O, Royo-Villanova Pérez ML. Relación entre maloclusión y disfunción de la ATM. Revisión bibliográfica. Rev Eur Odontoestomatol 1991;3:95-102.
22. Ramírez de Arellano ML, Torres JM, Rodríguez T. Posición condilar estable: ciexiste?, cies importante?. Rev Esp Ortod 2006;36:81-104.

23. Campos A. Rehabilitación oral y oclusal. Vol 1. Madrid: Ed Harcourt, SA; 2000.

24. Wood DP, Korne PH. Estimated and true hinge axis: a comparison of condylar displacements. Angle Orthod. 1992;62:167-75.

25. Alexander SR, Moore RN, Dubois LM. Mandibular condyle position: comparison of articular mounting and magnetic resonance imaging. Am J Orthod Dentofacial Orthop 1993;104:230-9.

26. Bujaldón Daza JM. Aplicación del análisis de cluster para definir grupos homogéneos desde el punto de vista de su posición condilar. Ortodoncia clínica 2000;3:32-35.

27. Cordray FE. Three-dimensional analysis of models articulated in the seated condylar position from a deprogrammed asymptomatic population: A prospective study. Part 1. Am J Orthod Dentofacial Orthop 2006;129:619-30.

28. Wood DP, Elliot RW. Reproducibility of the centric relation bite registration technique. Angle orthod 1994;64:211-20.

29. Karl PJ, Foley TF. The use of a desprogramming appliance to obtain centric relation records. Angle Orthod 1999; 69:117-23.

30. Hicks ST, Wood DP. Recording condylar movement with two facebow systems. Angle Orthod 1996; 66:293-300.

\section{CORRESPONDENCIA}

Gema Arroyo Cruz

Camacho, 54

21600 Valverde del Camino

Hueva

email: gemaac1@hotmail.com 